\title{
A Review of Researches Workplace Loneliness
}

\author{
Xuan Zhou \\ School of Management, Jinan University, Guangzhou, China \\ Email: zaosun@foxmail.com
}

How to cite this paper: Zhou, X. (2018). A Review of Researches Workplace Loneliness. Psychology, 9, 1005-1022. https://doi.org/10.4236/psych.2018.95064

Received: April 16, 2018

Accepted: May 15, 2018

Published: May 18, 2018

Copyright $\odot 2018$ by author and Scientific Research Publishing Inc. This work is licensed under the Creative Commons Attribution International License (CC BY 4.0).

http://creativecommons.org/licenses/by/4.0/

\begin{abstract}
Workplace health psychology is supported and upheld by psychologists, management scientists, sociologists and clinical psychology. Workplace loneliness has become a hot research topic in western occupational health psychology. The research about the spread of employee workplace loneliness in China is lagging behind. As a common negative emotion in workplace, workplace loneliness can bring in a series of negative effects on employees and individuals. At present in China, the transformation of the society and the rapid development of the economy have brought many problems to the workplace. To some extent, these problems will lead to the spreading of the common feeling of loneliness in the workplace. Therefore, it is necessary to study the crisis of workplace loneliness of our employees. At present, the study of loneliness in the workplace is still in its preliminary stage. The main purpose of this paper is to review the main subject of the study of loneliness in the workplace abroad, and to introduce its concepts, dimensions, measurement methods and related research into the country, and provide reference for Chinese scholars to study the rising crisis, that is, the sense of loneliness in the workplace.
\end{abstract}

\section{Keywords}

Loneliness, Interpersonal Relationship, Workplace

\section{Introduction}

According to previous studies of occupational health psychology, negative emotions in the workplace will bring a series of negative effects on employees and individuals. The sense of loneliness in the workplace is a common negative emotion in a workplace, and its negative emotions cannot be ignored. Especially, presently in China, we are in the stage of rapid industrialization and urbanization. The great changes in society and rapid economic development have brought up many questions to the workplace (Weiss, 1973). These problems will 
be in varying degrees. It leads to the spread of the sense of loneliness in the workplace. Firstly, the change in the method of working has made a lot of work possible to be done more independently, for example, For example, the division of labor between scientific and technological researchers is becoming more and more clear, and the opportunity for collaboration is declining gradually; a lot of independent and small consulting services or research and development design institutions have led many professional technicians and professionals work independently. Less and less work is done in a team or a collective way (Yengin Sarpkaya, 2014; Luo et al., 2012; Sayg et al., 2015). More and more employees have become isolated labor units, and it is difficult to have conditions or time to establish good interpersonal relationships in their work (Wright, 2007). The objective fact of this independent working system makes it difficult for many employees to feel the essence of being a part of a team or organization, and in a long period of isolation, it may induce their sense of loneliness in the workplace. Secondly, the presence of too many migrant workers in China at present, has led towards a gradual development of poor treatment towards them.

Human beings, as social animals, have social needs of social interaction and that of maintaining close relationships. If this need is not satisfied, the individual can easily experience the feeling of loneliness (Weiss, 1973). Loneliness is a basic/primitive experience of human beings. Few people can avoid this kind of experience in their life, especially with the advent of the age of information, the psychological distance between people is increasing, and the problem of loneliness is becoming more common (Wright, 2005a). Based on the universality of loneliness, a large number of scholars have made an empirical study of loneliness from the perspective of clinical psychology, and have achieved fruitful results ( $\&$ amp; nsal, 2014; Luo et al., 2012; Saygal, 2015). However, previous researches on loneliness mainly focused on personality factors, such as personality characteristics, and ignored environmental factors, especially the influence of working environment. Wright (2005a) pointed out that there are a variety of complex interpersonal relationships in the workplace, and it is easy to develop a sense of loneliness if the employees are unable to handle these relationships on a basic social level. Lam and Lau (2012) also point out that in the workplace, because of the popularity of network technology and the emergence of virtual teams, most employees pass information through the network media, the opportunities for face-to-face communication are reduced, and the fierce workplace competition makes the sincere contacts between the members of the organization difficult to maintain, and the effects of loneliness at workplace are highlighted (Lam \& Lau, 2012). Against this background, the concept of workplace loneliness has attracted the attention of scholars both at home and abroad. Loneliness in the workplace is an extension of the sense of loneliness in the field of organizational management (Tabancali, 2016). It will have an important impact on the working attitude, behavior and physical and mental health of the members of the organization. In terms of work attitude and behavioral performance, loneliness led to a decline in the performance of individual roles and a decrease in role behavior 
(Lam \&amp; Lau, 2012), and even the tendency to induce turnover (Ertosun \& amp; Erdil, 2012). In terms of physical and mental health, loneliness makes the individual's body and mind in subpar health condition, increasing the risk of physical health and the risk of anxiety and depression (Cacioppo et al., 2010). Based on the previous content, this paper reviews and summarizes the concept, structure and measurement of loneliness in the workplace, discusses its antecedent and consequence variables, and puts forward the integration model of loneliness in the workplace, and puts forward the prospect of the future research direction.

\section{Concept of Job Workplace Loneliness}

\subsection{Concept of General Loneliness}

Generally speaking, there are two views on the concept of general loneliness. Based on the theory of social needs (Weiss, 1973), we emphasize the emotional components of human need for intimacy and loneliness. For example, Weiss (1973) thinks loneliness is a kind of unpleasant and painful psychological feeling caused by the lack of intimacy and needs. Another view is based on social cognition theory, emphasizing individual's perception and evaluation of interpersonal relationship. For example, Peplau and Perlman (1982) regard loneliness as the perception and evaluation of individual's interpersonal relationship to reality in terms of quality and quantity. For everyone, there is an expectation level of social interaction in their minds, and it is easy to experience loneliness when the individual's real social interaction skill level is lower than that. Generally speaking, although there are certain differences in the above two views, they have their own unique application value: the first one is more suitable for the research in the field of clinical psychology, while the second view is applicable to the study of the loneliness of the general social masses (Peplau Perlman, 1982).

\subsection{Concept of Loneliness in the Workplace}

Compared with the general sense of loneliness, the concept of workplace loneliness has not yet been unified. A group of scholars believe that the sense of loneliness in the workplace is in essence consistent with the general sense of loneliness and the definition of the general loneliness is transferred directly to the workplace (Ayazlar \& Güzel, 2014); Ertosun \& amp; Erdil, 2012; Lam \& amp; Lau, 2012; Ozcelik \& Barsade, 2011). Another group of scholars, based on the situational features of the workplace, re defined the sense of loneliness in the workplace (Pinar, 2014; Silman \& amp; Dogan, 2013; Wright, 2005b; Yilmaz, 2011). For example, Wright (2005b) points out that there is a sense of loneliness in the workplace when there are differences in interpersonal relationships and interpersonal relationships that individuals expect in the workplace, and the lack of ability of the individuals to make up for this difference.

\subsection{Compare the General Loneliness and Workplace Loneliness}

There are differences and similarities between general loneliness and workplace 
loneliness.

The difference between these two is that the general sense of loneliness involves a wider range of relationships, including a variety of interpersonal relationships in daily life, but focusing on the relationship between the individual and friends and relatives; the sense of loneliness in the workplace is based on the working situation, is a psychological feeling or state, not a psychological trait, and is easily affected by the influence of the working situation (Wright, 2009). The change is focused on the relationship between the members of the organization (the leader and the followers), and the interpersonal relationship in the work is special. The working relationship is essentially a kind of economic interest based relation regulated by the formal rules and regulations. The space of individual free choice is relatively small and the deeper connection is loose or absent.

The similarities between the two: 1) The original reason is a kind of psychological perception triggered by the defect of interpersonal relationship; 2) In terms of characteristics, they all have subjective characteristics rather than objective characteristics in social activities (Yengin Sarpkaya, 2014); 3) The property valence is negative. It is often described as unpleasant, painful, sad, annoying and disgusting, etc.

Based on the above analysis, the sense of loneliness in the workplace is a kind of negative psychological feeling caused by the interpersonal relationship (social relationship and one on one relationship) in the workplace that cannot satisfy individual expectations in quantity or quality, and the individual lacks the ability to make up for this difference.

\subsection{The Different Concept between Workplace Loneliness and Other Loneliness}

\subsubsection{Solitude (Alone)}

Being alone is the objective state of a single person without or minimalistic interaction with the outside world. It can be regarded as an individual's choice or ability (Huang Yunzhen, Lin Shuhui, 2009). It should be emphasized that solitude is not necessarily accompanied by negative emotional experience. On the contrary, individuals who are alone may enjoy this process (Dussault \& Frenette. 2014). The sense of loneliness in the workplace is a subjective psychological feeling, not an individual's active choice, and a series of negative emotional experiences (such as pain and unhappiness). When an individual has to spend a long time alone non-voluntarily a sense of loneliness may develop (Long \&amp; Averill, 2003).

\subsubsection{Workplace Isolation}

Isolation in the workplace is a kind of cold violence in the workplace. It means that although individuals have the subjective intention to become members of the group, they are still crowded out of the organizational support network (Smith \& Calasanti, 2005). Workplace isolation is an objective phenomenon in 
an organization's social environment. It has passive features, and workplace loneliness emphasizes a subjective feeling of employees in the organization, both of which can cause great psychological stress and mental harm to individuals. Individuals are more difficult to change this kind of objective situation (Marshall et al., 2010). In general, scholars regard workplace isolation as an antecedent variable of workplace loneliness.

\subsubsection{Work Alienation}

The sense of work alienation means that the work situation cannot meet the needs of the employees. The disparity in the mental state of employees and work caused by disagreement (Banai et al., 2004). From the definition, it can be seen that the commonality of work alienation and workplace loneliness is that they are both subjective experiences and accompanied by negative emotions. The difference is: First, on the object, the sense of work alienation is reflected Employees' perception of the relationship between themselves and the work, while the loneliness side of the workplace Represent employees' perceptions of their relationship with members of other organizations. second, In terms of content, the sense of alienation of work is more extensive, including sense of powerlessness, No sense of sense, no sense of norms, sense of self-esteem and social isolation, and work Loneliness in a place only focuses on interpersonal issues in the workplace.

\section{The Structural Dimension of Loneliness in the Workplace}

The more popular views in the academic field about the structural dimension of loneliness are Russell's single dimension theory and Weiss's two element structure theory.

\subsection{Single Dimension}

Russell (1980) thinks loneliness is a single dimension emotional response, which is caused by the difference between the expectation and the actual level of social interaction (Russell et al., 1978). He believed that the core sense of loneliness is essentially the same. Lonely people feel and experience loneliness in the same way. Weiss (1987), on the other hand, puts forward a two structure on the basis of the above views, and further divides the loneliness into emotional loneliness and social loneliness (Russell et al., 1980; Russell, 1996). Emotional loneliness refers to the loneliness caused by the failure to meet the needs of the individual, and the social loneliness means that the individual is not satisfied with the needs of its social integration (Ozcelik \& Barsade, 2011; Lam \& Lau, 2012).

Among them, the workplace loneliness scale compiled by Wright (2005b) is more mature and widely used. Some researchers modified the off-the-shelf UCLA Loneliness Scale (Russell, 1980) to measure workplace independence by using "work in progress" as a precursor to each project (Cubitt \& Burt, 2002; Dussault \& Thibodeau, 1997). Other researchers use direct questioning to measure loneliness in the organization (Bell, Roloff, Camp \& Karol, 1991; Reinking \& Bell, 1991). 


\subsection{Two Dimension}

The workplace sense of loneliness measured by this method is one-dimensional and it is overall loneliness. In one study, Ozcelik conducted a measurement of loneliness in the workplace based on the adapted UCLA Loneliness Scale, and the results obtained were consistent with the results of group assessments (Ozcelik, 2011). Although group assessment is an objective method, it measures the sense of loneliness in the workplace as a single dimension and does not accurately reflect the content and structure of loneliness in the workplace. Among them, the workplace loneliness scale compiled by Wright (2005a) is more mature and widely used. Some researchers modified the off-the-shelf UCLA Loneliness Scale (Russell, 1980) to measure workplace independence by using "work in progress" as a precursor to each project (Cubitt \& Burt, 2002; Dussault \& Thibodeau, 1997). Other researchers use direct questioning to measure loneliness in the organization (Bell, Roloff, Camp \& Karol, 1991; Reinking \& Bell, 1991).

These questionnaires based on the single-dimension theory do not consider the individual's loneliness in any circumstances, and cannot distinguish the source of loneliness. The measurement method based on the dual structure theory is widely recognized, among which Wright's workplace loneliness questionnaire is the most famous. Based on literature analysis and theoretical analysis, Wright (2005b) borrowed UCLA Loneliness Scale, State-Special Loneliness Scale, Emotional Loneliness, and Social Loneliness Scale to compile a multi-dimensional workplace loneliness scale including emotions. Deprivation of 7 topics and lack of 7 memberships in the organization (Wright et al., 2006). The alpha coefficient of the actual report total table is .94, and the test-retest reliability of the total table is .80 . The weight of the emotional deprivation subscale and organizational membership subscale is deducted. The degree of confidence was all .83 , and the cumulative variance contribution rate was $61.81 \%$. Wright revised this scale in 2006 and called the Loneliness at Work Scale. It has a good reliability and validity and is widely used.

In the context of our country's culture, Mao Zhonglin (2013), referring to the results of previous studies, adopted in-depth interviews and open questionnaires to compile the workplace loneliness scale. The scale includes five problems of existential loneliness and five problems of interpersonal loneliness. The alpha coefficient of the actually reported total table is .88 , and the alpha coefficient of the subscale is above 0.85 . The scale has good content validity and structural validity. Due to the rarity of empirical studies on loneliness in the workplace, this scale has not been fully validated.

\section{Methods to Measure Job Search Clarity}

There are mainly two types of measures in the West,: group assessment methods and questionnaire surveys. The workplace loneliness measured by this method is a single dimension and a general sense of loneliness. In one study, Hakan.Ozcelik (2011) measured the workplace loneliness based on the revised UCLA loneliness 
scale, and the results were consistent with the results of group assessment. Although group assessment is an objective method, the sense of loneliness in the workplace is a single dimension, which cannot accurately reflect the content and structure of the sense of loneliness in the workplace. Therefore, more scholars tend to develop a suitable scale as a measuring tool. Among them, the Wright (2005a) workplace loneliness scale is relatively mature and widely used. Some researchers modified the ready-made UCLA Loneliness Scale (Russell, 1980) to measure workplace loneliness (Cubitt \&amp; Burt, 2002; Dussault \&amp; Thibodeau, 1997) by using "work" as the leader of each project. Other researchers used direct questioning to measure loneliness in the organization (Bell, Roloff, Camp \& Karol, 1991; Reinking \&amp; Bell, 1991).

These questionnaires based on the single dimension do not consider the individual's loneliness under any circumstances, and can't distinguish the source of loneliness. The measurement method based on the two element structure theory has been generally recognized. The Wright workplace Loneliness Questionnaire is the most famous. Wright (2005b) based on the literature analysis and theoretical analysis, using the UCLA loneliness scale, state trait loneliness scale, emotional loneliness and social loneliness scale, the multi-dimensional workplace loneliness scale was compiled, including 9 topics of emotional deprivation, 7 missing titles of organization members, and the total amount of measured reports. The coefficient of alpha was 94 , the retest reliability of the total scale was .80 , and the retest reliability of the emotional deprivation scale and the organization members' identity missing subscale was 83 , and the cumulative variance contribution rate was $61.81 \%$. Wright revised this scale in 2006. It is called LAWS at (Loneliness Scale). It has good reliability and validity and is widely used.

Under the cultural background of China, Mao Lin (2013) referred to the results of previous studies, and adopted in-depth interviews and open questionnaire surveys to compile the workplace loneliness scale. The scale includes 5 topics of sexual loneliness and 5 topics of interpersonal loneliness. The alpha coefficient of the total amount of measured reports is.88, and the alpha coefficient of the subscale is above .85 . The scale has good content validity and construct validity. Due to the rare empirical research on workplace loneliness in China, this scale has not yet been fully verified.

\section{Influencing Factors of Workplace Loneliness}

In the workplace, a series of factors such as personality, social intelligence, work values, working hours, work load, organizational climate, organizational support and human organization matching will not only affect the loneliness of the followers, but also affect the loneliness of the leaders, that is, the common antecedents of the two. However, because leaders and followers are different in rank, they also have unique influence factors. Therefore, this paper first explores the common antecedents of loneliness and the loneliness of the followers from three aspects of individual characteristics, work characteristics and organizational 
characteristics, and then sort out the unique antecedents of the two.

\subsection{Individual Characteristics}

\subsubsection{Personality}

Personality, as a relatively stable individual characteristic, plays an important role in the formation of workplace loneliness. The influence of extraversion and emotional stability, shyness, social avoidance and competitive attitude on loneliness in the workplace has been confirmed by researchers in the five personality (Mao Zhonglin, 2013; Wright, 2005a). In particular, extraversion and emotional stability have a significant negative correlation with workplace loneliness, and the more extroverted and emotionally stable employees, the more able to establish good interpersonal relationships with others. Shyness reflects the shortcomings of employees' social skills. Social avoidance can reflect employees' low social intercourse. They can positively predict workplace loneliness. An individual with a strong sense of competition is eager to win and surpass others, and it is difficult to trust and form a close relationship with others. Therefore, the competitive mentality is positively related to the sense of loneliness in the workplace.

\subsubsection{Social Intelligence}

Social intelligence (Social Intelligence) is an important indicator of whether individuals can handle good interpersonal relationships, and loneliness is caused by the defects of interpersonal relationships, and they have a certain relationship. Silman and Dogan (2013) study found that the higher the social intelligence scores, the less the sense of loneliness experienced by the employees; the three dimensions of social intelligence, the social information processing (social information processing), the social skills (social skills) and the social awareness (social awareness), to predict the loneliness of the workplace. In terms of emotional deprivation dimension, social skills and social awareness negatively predict the social dimension of workplace loneliness.

\subsubsection{Working Values}

Work value is the value orientation that individuals show in their professional life, and it is also the individual's expectation to get satisfying needs from work (Yilmaz, 2011). Yilmaz (2011) was investigated using the SVI (Schwartz Values Inventory) value scale. The results showed that employees scored higher on the four dimensions of self transcendence, self improvement, traditionalism and the degree of openness to change, the less the sense of loneliness in the workplace, and when employees had similar values with other organization members. They tend to engage in more communication and interaction. On the contrary, when values are different, employees can not establish and maintain high quality relationships with their members.

\subsection{Working Characteristics}

In the workplace, job characteristics have a certain impact on loneliness. Bell et al. (1991) found that working hours can effectively predict the loneliness of 
members. The longer working hours, the easier employees are to ignore interpersonal relationships, and the more likely they are to experience loneliness. This relationship is regulated by team cohesion (work-group cohesion), that is, when the team cohesion is high, the relationship between job length and loneliness is not significant; and when the cohesiveness of the team is low, there is a strong positive correlation. Besides, there is a significant positive correlation between work load and workplace loneliness, too much work load, fatigue, burnout, and lack of time and energy to deal with problems in interpersonal relationships, and then experience loneliness (Wright, 2005b).

\subsection{Organizational Characteristics}

\subsubsection{The Atmosphere of the Organization}

Organizational climate is an employee's perception of the quality of the internal environment of an organization, and plays an important role in the formation and development of interpersonal relationship in work (Wright, 2005a). The empirical investigation found that the atmosphere of solidarity (community Spirit) has a significant negative correlation with the workplace loneliness, and the climate of fear has a significant positive correlation with the workplace loneliness. When organizational climate emphasizes rewards and penalties and personal interests, while ignoring team cooperation and mutual trust, it is difficult to form a friendship in the workplace (Wright, 2005b). Mao Zhonglin's (2013) study further indicated that warm support, job autonomy, reward orientation and management efficiency have significant negative explanatory power on workplace loneliness, and warm support is the intermediary variable between personality variables and workplace loneliness.

The study of Erdil and Ertosun (2011) also found that interpersonal atmosphere is an important antecedent variable of the sense of loneliness at the organizational level, the more the interpersonal atmosphere in the organization supports positive interpersonal relationships, the less the sense of loneliness in the workplace is experienced by the employees.

\subsubsection{Organization Support}

The lack of organizational support is an important inducement of the sense of loneliness in the workplace. The lack of support staff can only rely on themselves to solve problems in their work, less experience of other people's care and help, and it is difficult to form intimate relationships and ownership.

Sense. Empirical research shows that superior support and peer support significantly negatively predict workplace loneliness (Wright, 2005a). Stoica et al. (2014) further pointed out that leaders' timely feedback and recognition on their followers' work is an important way to reduce their loneliness. In addition, a study focused on the loneliness of leaders found that colleagues supported a stronger sense of loneliness than the support of superior support and followers (Greene, 2016). The equality of their positions made the leaders more inclined to adopt self-exposure strategies in the process of interacting with their colleagues 
so that they were under pressure. Support was obtained to reduce workplace loneliness (Lindorff, 2001).

\section{Influencing Results of Workplace Loneliness}

Workplace loneliness hinders effective communication and interaction among members, and causes a series of negative effects on themselves, teams and organizations. This paper discusses the consequences of workplace loneliness from four aspects: work attitude, work behavior, mental health and physical health.

\subsection{Working Attitude}

\subsubsection{Job Satisfaction and Happiness}

The influence of employees' job satisfaction and happiness on workplace loneliness. The empirical study showed that workplace loneliness was negatively related to job satisfaction (Wright, 2005a), and had a negative correlation with job happiness (Erdil \& Ertosun, 2011). Job satisfaction and happiness are a positive emotional response to the job, and the sense of loneliness in the workplace is a negative emotional experience, which is avoided by the members of the organization and is not welcomed by the members of the organization (Ozcelik \& Barsade, 2018). If the employee's interpersonal relationship in the workplace is defective in quantity or quality and does not reach the expected expectations, the employee's positive emotional response to work will be reduced, which leads to a decline in job satisfaction and happiness (Lam \& Lau, 2012). However, a survey of loneliness of migrant workers in China shows that lonely workers are more satisfied with their job satisfaction (Chan \& Qiu, 2011), which indicates that there may be a regulatory variable between loneliness and job satisfaction in the workplace.

\subsubsection{Organizational Commitment and Turnover Intention}

If the organization is unable to meet the staff, as for the needs of intimacy and social relationships, employees' organizational commitment will be reduced, which leads to an increase in the possibility of leaving the present organization to find more satisfactory interpersonal relationships. Wright (2005a) found that workplace loneliness was negatively correlated with organizational commitment and positively related to turnover intention. Ertosun and Erdil (2012) use Wright's LAWS scale to investigate the impact of loneliness in the workplace on organizational commitment and turnover intention. It is found that emotional deprivation and social loss are positively related to turnover intention. Both can positively predict turnover intention; emotional deprivation and social lack are negatively related to emotional commitment and social lack. Loss can negatively predict affective commitment, and affective commitment is a mediator between social loss and turnover intention. Ayazlar and Guzel (2014) also found that workplace loneliness negatively predicted organizational commitment. Compared with emotional deprivation, social lack has a stronger explanatory power to organizational commitment. However, Chan and Qiu (2011) research shows 
that loneliness and organizational commitment are significant for Chinese migrant workers.

\subsection{Work Behavior}

\subsubsection{Job Performance}

Ozcelik and Barsade (2011) explained the relationship between loneliness and job performance in the workplace through two mechanisms.

From the point of attention and cognitive mechanism, employees because of the loneliness in the workplace, afraid of being found out, want to get rid of the negative emotions of self-adjusting efforts will take up a lot of cognitive resources, and led to a decline in performance, namely the workplace loneliness play a vital role in reducing the task performance by increasing the employee's stress surface.

From the point of relationship mechanism, when employees feel more distant and other members of the organization and due to the lack of contact, the individual will lack the sense of belonging to an organization, reduce their emotional commitment to the organization, resulting in a decline in performance, namely the workplace loneliness by reducing the emotional commitment to reduce task performance of employees.

In addition, the study also found that the relationship between individual performance in a team and an overall team performance is also influenced by the workplace loneliness, often experience the loneliness of employee relations in the workplace performance and team performance is low.

This shows that the loneliness in the workplace is not only a personal problem for employees, but also a problem of team and organization. The loneliness of the members of the organization can easily affect others.

The members, who in turn, hinder the effectiveness of the team and the organization.

\subsubsection{Organizational Citizenship Behavior}

Lam and Lau (2012) point out that workplace loneliness is negatively correlated with organizational citizenship behavior. Based on social exchange theory, an individual hopes to establish a stable trustworthy relationship with others in order to achieve a long-term reciprocity. If the organization meets the needs of intimate relationships and social contacts, employees will not only do their job well, but also pay extra efforts to achieve organizational goals (Lam \& Lau, 2012). For lonely employees, employees are unwilling to undertake social exchange with organizations because of their negative cognition towards themselves and lack of trust in others.

Risk and reduce altruistic behavior in the organization.

\subsubsection{Leader Member Exchange (LMX) and Organizational Member Exchange (OMX)}

The LMX theory holds that leaders will establish varying degrees of exchange relationship in the process of interaction with their followers (Graen Uhl-Bien 
\&amp; 1995). In low quality LMX, there is only economic exchange between leaders and followers; in high quality LMX, there is emotional exchange between the two, which shows that the leaders have more respect, trust, love and responsibility for the followers, and the followers will be rewarded with excellent job performance and frequent organizational citizenship behavior (Christie \& Geis, 1973). The study of Lam and Lau (2012) showed that the sense of loneliness in the workplace was negatively correlated with LMX, and LMX played a mediating role in the negative relationship between loneliness and organizational citizenship behavior in the workplace. On the one hand, the followers with higher loneliness have a negative evaluation of others, are reluctant to communicate with the leaders and are afraid of the risk of social exchange (Peng, Chen, Xia, \& Ran, 2017); on the other hand, because of their negative and negative evaluation of themselves, they believe that they lack the power of the leaders' requirements and are reluctant to accept the angle of the leaders' distribution. Color tasks, so it is difficult to establish high quality (Lindorff, 2001).

LMX, and refuse to make extra efforts for leaders, so as to reduce organizational citizenship behavior. Besides exchanging social relations with leaders, followers can also exchange with organizations (Öge, Çetin, \& Top, 2018). Since the organization is more distant than a specific individual, the higher solitude followers believe that there is a higher risk of social exchange and a low quality OMX. In this case, the followers feel less organizational support and resource allocation, and are less likely to complete. A good job. Therefore, workplace loneliness is negatively correlated with OMX, while OMX is negatively correlated with workplace loneliness and role performance (Lam \&amp; Lau, 2012).

\subsection{Mental Health}

Loneliness is a painful psychological experience, which can lead to a series of mental health problems. For example, the sense of loneliness in the workplace significantly increased the job stress of the employees, and the employees who did not experience loneliness were more effective in dealing with stress events (Wright, 2005b). From an evolutionary point of view, loneliness is not only an unpleasant experience, but also the insecurity of the surrounding environment, which leads to unpleasant feelings such as anxiety, fear and low self-esteem (Cacioppo et al., 2014). There are also studies that show that loneliness and depression are inseparable. Lonely individuals often form negative perceptions of themselves (Rokach, 2012), often feeling sad, dissatisfied and helpless, and are more pessimistic in looking at things (Mushtaq et al., 2014). Loneliness has great damage to the individual's cognitive ability, which reduces the memory and reaction speed of the person, and can also lead to personality disorders such as marginal personality disorder and schizophrenia (Mushtaq et al., 2014).

\subsection{Physical Health}

Loneliness is not only a psychological problem, but also a physiological problem. 
Due to the universality of loneliness, the physical damage of the employees due to loneliness cannot be ignored. A large number of studies have shown that loneliness endangers the health of people, resulting in poor sleep quality, fatigue (Robinson Marentette, 2014) and elevated blood pressure (Hawkley et al., 2010). The long and serious loneliness has not been alleviated and the immune system, cardio cerebral blood tube and other diseases are caused, and suicide and cancer are increased. The probability (Mushtaq et al., 2014). A tracking survey shows that loneliness can effectively predict life expectancy, and the life expectancy of people experiencing less loneliness is significantly higher than that of people who often experience loneliness (Stessman et al., 2014). The results of a meta-analysis also showed that the negative impact of loneliness on life expectancy was no less than known risk factors, such as smoking and air pollution (Holt-Lunstad et al., 2015).

\section{Conclusion and Prospect}

\subsection{Conclusion}

Many practitioners engaged in human resources work are very interested in the sense of loneliness in the workplace. They are accustomed to using "workplace autism" to define this kind of negative sentiment spreading in the workplace. In short, the reality of the workplace and the actual needs of management all require that our academic community must give more attention and study to the sense of loneliness in the workplace. The study of loneliness in the workplace in foreign countries is also in the rising stage. Its research results are still not abundant and do not form a complete theoretical system.

Firstly, in the study of loneliness in the workplace, there are not many empirical studies on workplace loneliness from the perspective of organizational climate. Although many scholars believe that the organizational climate is an important factor influencing loneliness in the workplace, only a few scholars have examined in detail the relationship between organizational climate and workplace loneliness. Moreover, the dimensionality of the organizational climate has been very rough. From the perspective of Lewin's group dynamics and man-environment matching theory, the sense of loneliness in the workplace is generated during the interaction between employees and organizations. The generation of loneliness in the workplace is examined from the perspective of organizational climate. Matching research trends, future research should examine the relationship between organizational dimensions and workplace loneliness.

Second, there is a lack of research on the effect of loneliness in the workplace on outcome variables. Most studies only discuss the correlation between the two. Only Öya Erdil and Öznur Gülen·Ertosun (2012) found that the sense of loneliness in the workplace influences employee turnover tendencies (supplementary new ones) through the mediating role of organizational commitment. In the future research, examining the influence mechanism of loneliness in the workplace on outcome variables should be the focus of research. 
Thirdly, there is still controversy on the separation of influence variables and outcome variables in the sense of loneliness in the workplace. Some variables and the sense of workplace independence may influence each other, such as job burnout and workplace loneliness. Some studies believe that job burnout induces workplace.

Loneliness, but also some scholars believe that workplace loneliness is the cause of job burnout (Pines \& Aronson, 1988). The relationship between many variables and loneliness in the workplace may not be as simple as impacts and impacts. The relationship between the two may be mutually influenced. In future studies, special attention should be paid to not arbitrarily drawing on the basis of detailed data analysis.

\subsection{Research Prospects}

\subsubsection{Localization Measurement}

The existing workplace loneliness scale is mainly the LAWS scale compiled by Wright et al. (2006). The scale has been repeatedly verified by foreign scholars and has a good reliability and validity. However, the culture may be used in China's cultural context. Adaptability issues. Mao Zhong lin (2013) proposed in his research that because of the different cultures, the sense of loneliness in the workplace of both East and West has both commonalities and characteristics. Therefore, in the future, localization of loneliness in the workplace should be studied to develop a scale suitable for loneliness in the workplace in China, and to provide an effective measurement tool for follow-up research by Chinese scholars.

\subsubsection{Multiple Measurement Method}

In addition, previous researches mostly use self-assessment of employees to study loneliness, which has some shortcomings. For example, people usually do not want to express their past or present lonely experiences, and many people are ashamed to acknowledge the fact that it is a social failure (Ertosun Erdil, 2012).

The employee self-assessment is difficult to avoid the social desirability bias, the results may not be able to reflect the psychological state of employees. But the subjective feeling of loneliness makes it difficult for him to evaluate the true feelings of the evaluator accurately. The study of Ozcelik and Barsade (2011) combines two ways of self-evaluation and evaluation, which is worth learning from. The self-assessment can reflect the difference between the actual interpersonal relationship and the expectation level, and reveal the quality of the interpersonal relationship in the workplace, and he can reflect the number of interpersonal relationships in the individual workplace. And, in addition to use

Besides measuring scale, loneliness can also be manipulated by experiment (Snyder, 2014). Loneliness can also be performed experimentally (Snyder, 2014). The future research can be self-evaluation and he commented on the two scales measurement methods. For example, Figure 1 is the object of the followers to 


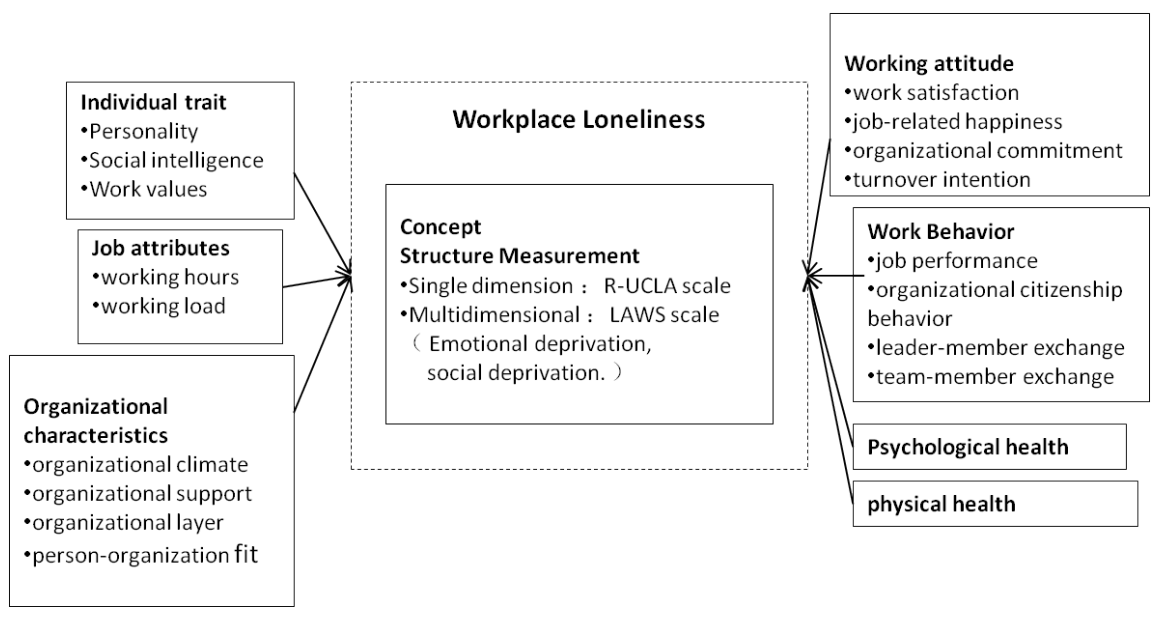

Figure 1. Integration model of workplace loneliness.

imitate (Li, Ye, Management, \& University, 2015). The followers tend to learn from the leaders and pay more attention to the leaders' emotions. Therefore, the leaders' emotions may be transmitted to the followers from top to bottom. Future research can explore the loneliness of leaders Line transfer effect.

\subsubsection{Matching Research}

Match between leaders and their followers in recent years. (Leader-Follower congruence) has gradually become a hot topic in the study of organizational behavior. At present, scholars mainly focus on the positive psychological matching between leaders and followers (Zhang et al., 2012; Landry et al., 2014), and the matching of negative psychology is still rare. In case of the sense of loneliness, it is also possible that there is a matching effect between leaders and followers. Similar attraction theory (similarity attraction effect) holds that in the process of interpersonal communication, there are similar characteristics (such as demographic variables, personality, etc.) Individuals are prone to attract each other and show more willingness to communicate and interact; on the contrary, disparate individuals are prone to rejection and antipathy (Byrne, 1971). In the organization, if the followers and the leaders experience the same sense of loneliness, they have a relatively consistent view of the quality of the interpersonal relationship in the workplace (satisfaction or dissatisfaction). The similarity of this attitude makes it possible for leaders and followers to attract and love each other and bring a series of positive results, such as the improvement of the LMX (Kacmar et al., 2009).

Mood congruency effect believes that individuals can make a tendency to judge according to their own emotional state. Individuals in the state of positive emotion are more likely to recall positive things and make positive judgments; on the contrary, individuals in the state of negative emotions are more likely to recall negative ones. Things, and make a negative judgment (Bodenhausen et al., 1994). Loneliness, as a negative psychological state, when it is inconsistent between the leader and the followers, the party experiencing more loneliness can 
easily have a more negative evaluation of the other side and even the negative behavior, while the side with lesser loneliness is relatively less negative and relatively less negative behavior. This is not the case. It may bring some negative consequences, such as the reduction of LMX. Therefore, future research can explore the influence mechanism of loneliness from the perspective of leader follower's loneliness matching.

\subsubsection{Multilevel Research}

At present, the research on workplace loneliness is focused on individual level, but there is a lack of research on team level and organizational level. Workplace loneliness is not only affected by employees' characteristics, emotion and cognition, but also by team and organizational factors. Therefore, the follow-up study can introduce more team or organizational variables to further clarify the organizational and boundary conditions of the sense of loneliness in the workplace. In addition, the loneliness of the members of the organization can be aggregated to the team level to explore the effect of the loneliness atmosphere on the individual or organizational level.

\subsubsection{Short Term Fluctuation}

As a psychological state, workplace loneliness is sensitive to the external environment, and is easy to be affected by the working situation. From this dynamic perspective, it is of great theoretical and practical significance to study the short-term internal fluctuations of workplace loneliness. The existing research focuses on cross section design. From the perspective of the individual, it explores the causes, consequences and mechanisms of loneliness in the workplace, that is, why different employees experience different degrees of loneliness, but this has largely obscured the dynamic and state characteristics of the sense of loneliness in the workplace. In fact, the level of loneliness in individual employees varies with the working environment, and the same employee experiences a different degree of loneliness at different times, the point which needs to be stressed.

\section{References}

Ayazlar, G., \& Güzel, B. (2014). The Effect of Loneliness in the Workplace on Organizational Commitment. Procedia - Social and Behavioral Sciences, 131, 319-325. https://doi.org/10.1016/j.sbspro.2014.04.124

Banai, M., Reisel, W. D., \& Probst, T. M. (2004). A Managerial and Personal Control Model: Predictions of Work Alienation and Organizational Commitment in Hungary. Journal of International Management, 10, 375-392. https://doi.org/10.1016/j.intman.2004.05.002

Bell, R. A., Roloff, M. E., Camp, K. V., \& Karol, S. H. (1991). Is It Lonely at the Top?: Career Success and Personal Relationships. Journal of Communication, 40, 9-23. https://doi.org/10.1111/j.1460-2466.1990.tb02247.x

Bodenhausen, G. V., Sheppard, L. A., \& Kramer, G. P. (1994). Negative Affect and Social Judgment: The Differential Impact of Anger and Sadness. European Journal of Social 
Psychology, 24, 45-62. https://doi.org/10.1002/ejsp.2420240104

Cacioppo, J. T., Cacioppo, S., \& Boomsma, D. I. (2014). Evolutionary Mechanisms for Loneliness. Cognition \& Emotion, 28, 3-21. https://doi.org/10.1080/02699931.2013.837379

Cacioppo, J. T., Hawkley, L. C., \& Thisted, R. A. (2010). Perceived Social Isolation Makes Me Sad: 5-Year Cross-Lagged Analyses of Loneliness and Depressive Symptomatology in the Chicago Health, Aging, and Social Relations Study. Psychology and Aging, 25, 453-463. https://doi.org/10.1037/a0017216

Chan, S. H., \& Qiu, H. H. (2011). Loneliness, Job Satisfaction, and Organizational Commitment of Migrant Workers: Empirical Evidence from China. International Journal of Human Resource Management, 22, 1109-1127. https://doi.org/10.1080/09585192.2011.556785

Christie, R., \& Geis, F. L. (1973). Studies in Machiavellianism. American Political Science Association, 67, 400-407.

Dussault, M., \& Frenette, É. (2014). Loneliness and Bullying in the Workplace. American Journal of Applied Psychology.

Erdil, O., \& Ertosun, Ö. G. (2011). The Relationship between Social Climate and Loneliness in the Workplace and Effects on Employee Well-Being. Procedia-Social and Behavioral Sciences, 24, 505-525. https://doi.org/10.1016/j.sbspro.2011.09.091

Greene, N. T. (2016). Loneliness and Perceived Social Support in the Workplace of the School Principal. Proquest LLC.

Lam, L. W., \& Lau, D. C. (2012). Feeling Lonely at Work: Investigating the Consequences of Unsatisfactory Workplace Relationships. International Journal of Human Resource Management, 23, 4265-4282. https://doi.org/10.1080/09585192.2012.665070

Li, C., Ye, M., Management, S. O., \& University, J. (2015). Review on Workplace Loneliness. Human Resources Development of China.

Marshall, G. W., Michaels, C. E., \& Mulki, J. P. (2010). Workplace Isolation: Exploring the Construct and Its Measurement. Psychology \& Marketing, 24, 195-223.

https://doi.org/10.1002/mar.20158

Mushtaq, R., Shoib, S., Shah, T., \& Mushtaq, S. (2014). Relationship between Loneliness, Psychiatric Disorders and Physical Health ? A Review on the Psychological Aspects of Loneliness. Journal of Clinical \& Diagnostic Research, 8, 1-4.

Öge, E., Çetin, M., \& Top, S. (2018). The Effects of Paternalistic Leadership on Workplace Loneliness, Work Family Conflict and Work Engagement among Air Traffic Controllers in Turkey. Journal of Air Transport Management, 66, 25-35. https://doi.org/10.1016/j.jairtraman.2017.10.003

Ozcelik, H., \& Barsade, S. (2011). Work Loneliness and Employee Performance. Academy of Management Annual Meeting Proceedings, 2011, 1-6. https://doi.org/10.5465/ambpp.2011.65869714

Ozcelik, H., \& Barsade, S. (2018). No Employee an Island: Workplace Loneliness and Job Performance. Academy of Management Journal, 2015, 1066. https://doi.org/10.5465/amj.2015.1066

Peng, J., Chen, Y., Xia, Y., \& Ran, Y. (2017). Workplace Loneliness, Leader-Member Exchange and Creativity: The Cross-Level Moderating Role of Leader Compassion. Personality \& Individual Differences, 104, 510-515. https://doi.org/10.1016/j.paid.2016.09.020

Reinking, K., \& Bell, R. A. (1991). Relationships among Loneliness, Communication Competence, and Career Success in a State Bureaucracy: A Field Study of the "Lonely 
at the Top" Maxim. Communication Quarterly, 39, 358-373. https://doi.org/10.1080/01463379109369812

Rokach, A. (2012). Leadership and Loneliness: A Review. Psychology Journal.

Stoica, M., Brate, A. T., Bucuța, M., Dura, H., \& Morar, S. (2014). The Association of Loneliness at the Workplace with Organisational Variables. European Journal of Science \& Theology, 10, 101-112.

Tabancali, E. (2016). The Relationship between Teachers' Job Satisfaction and Loneliness at the Workplace. Eurasian Journal of Educational Research, 16, 1-30. https://doi.org/10.14689/ejer.2016.66.15

Wright, S. (2007). The Experience of Loneliness in Organisations. Christchurch: University of Canterbury Management.

Wright, S. L. (2005a). Loneliness in the Workplace (Vol. 1, pp. 123-142). VDM Verlag Dr. Müller Aktiengesellschaft \& Co. kg.

Wright, S. L. (2005b). Organizational Climate, Social Support and Loneliness in the Workplace. Research on Emotion in Organizations, 1, 123-142. https://doi.org/10.1016/S1746-9791(05)01106-5

Wright, S. L. (2009). In a Lonely Place: The Experience of Loneliness in the Workplace.

Wright, S. L., Burt, C. D. B., \& Strongman, K. T. (2006). Loneliness in the Workplace: Construct Definition and Scale Development. New Zealand Journal of Psychology, 35, 59-68.

Yengin Sarpkaya, P. (2014). The Effects of Principals' Loneliness in the Workplace on Their Self-Performance. Educational Research \& Reviews, 9, 967-974. https://doi.org/10.5897/ERR2014.1847

Yilmaz, E. (2011). An Investigation of Teachers' Loneliness in the Workplace in Terms of Human Values They Possess. African Journal of Business Management, 5, 5070-5075. 